\author{
Marta Woźnicka*
}

\title{
DIE FORMALE ENTWICKLUNG DES VERBS TUN IM DEUTSCHEN. EINE KORPUSBASIERTE UNTERSUCHUNG VOM ALT- BIS ZUM FRÜHNEUHOCHDEUTSCHEN
}

\section{Herkunft, Bedeutung und Funktion von tun}

Das Verb tun wird zur Gruppe der Verben mit besonderer Flexion gerechnet, die sie von zwei Hauptklassen der deutschen Verben, d. i. den starken und schwachen Verben unterscheidet, und es gehört samt Verben sein, gehen, stehen zu sog. athematischen Verben ggf. Wurzelverben, die ursprünglich im Stamm keinen Themavokal aufwiesen und in der 1. P. Sg. die primäre Endung -mi zeigten (gehen, stehen, tun) bzw. nach wie vor zeigen (sein): bi-n (Braune, Reiffenstein 2004, S. 309 311; Paul 2007, S. 275-276; Ebert, Reichmann, Solms, Wegera 1993, S. 305-306). Tun ist ausschließlich in westgermanischen Dialekten erhalten: Die rekonstruierte indogermanische Form*dhe-, germanische *dō- entwickelt sich im 8. Jhd. zu tuon (Althochdeutsch) und don (Altenglisch) (Seebold 1970, S. 157-159; Lühr 1984, S. 39-40; Bammesberger 1986, S. 112-114; Matzel 1987, S. 171-172).

In Grammatiken zum Gegenwartsdeutschen wird das Verb tun ausführlich behandelt. Als Vollverb charakterisiert das Verb eine Handlung oder Tätigkeit: Er tat, was ihm befohlen wurde, Sie tut ihre Arbeit. Im Satz Er tut so, als ob er angle wird eine vorgetäuschte Aktivität ausgedrückt. Im zusammengesetzten Satz kann dagegen tun das Verb oder die ganze Verbalphrase ersetzen: Ich riet ihr zu verschwinden, was sie auch schleunigst tat ${ }^{1}$.

Das Verb tun, wie auch andere Verben mit archetypischer Bedeutung, wird von Grammatikalisierungsprozessen betroffen.

Laut einer Hypothese, die von Diederich von Stade bereits im Jahre 1718 formuliert worden ist und die als Kompositionstheorie (Collitz 1888, S. 42) oder

* Dr. habil. Marta Woźnicka, Institut für germanische Philologie der Adam-Mickiewicz-Universität in Poznań, al. Niepodległości 4, 61-874 Poznań. E-Mail: marado@amu.edu.pl.

${ }^{1}$ Die Beispielsätze entnommen aus: DUDEN. Das große Wörterbuch der deutschen Sprache 1999, S. 3993-3995. 
Agglutinations-Hypothese (Hirt 1927, S. 4) bezeichnet wird, stammt das germanische *-đ- /ठ/ (hochdeutsch - $t$-) des Präteritums der schwachen Verben von dem germanischen Verbalstamm *dhê-/*dhô- ,tun', das an den entsprechenden Verbalstamm angeschlossen wird und allmählich zum grammatischen Element geworden ist (Collitz 1912, S. 1). Diese Hypothese galt längere Zeit als unumstritten und wurde u. a. von Bopp (1816, S. 151-153), Grimm (1/1898/1989, S. 563), Hirt (1927, S. 4), Sverdrup (1929, S. 5-7) geteilt ${ }^{2}$.

Der nächste Grammatikalisierungsprozess betrifft die Entwicklung von tun zum Auxiliarverb. Die Periphrase mit dem Verb tun als dem Hilfs- bzw. lediglich dem infinitivregierenden Verb (DUDEN. Die Grammatik 2005, S. 423) wird in Grammatiken zum Gegenwartsdeutschen als umgangssprachlich und im Standarddeutschen als ungrammatisch bewertet, z. B. Ich tue essen (DUDEN. Die Grammatik 2001, S. 667), obwohl die Variante mit dem Vollverb im Vorfeld des Satzes, z. B. Schreiben tue ich ihm nicht (Eichinger 2014, S. 104) oder Sehen tu ich nichts (DUDEN. Die Grammatik 2005, S. 422) als der Rhematisierung dienende Konstruktion betrachtet wird (DUDEN. Die Grammatik 2001, S. 667; Eichinger 2014, S. 104; Deuber et al. 2007). Auch nach Engel (1996, S. 476) dient die tun-Periphrase der Hervorhebung des Vollverbs und wird in der Alltags- und Kindersprache vorwiegend mit dialektaler Färbung in Mittel- und Norddeutschland verwendet, obwohl das Phänomen eher dem Süden zugeschrieben wird. Nach Deuber (2007) werden durch tun nur synthetische Tempusformen ergänzt, d. i. Präsens Indikativ (er tut schreiben), Präteritum Indikativ (er tat schreiben), Präteritum Konjunktiv (er täte schreiben) und Imperativ (Tu schreiben!). Analytische Tempora können derart nicht umschrieben werden.

In verschiedenen Entwicklungsetappen des Deutschen sind Grammatikalisierungsprozesse vom Verb tun zum Hilfsverb sichtbar: Weiss (1956) beschreibt die Anfänge und die Entwicklung der tun-Periphrase vom Alt- bis zum Mittelhochdeutschen (bis um 1400); Langer (2001) konzentriert sich dagegen auf ihre Blütezeit und ihr Ende im Frühneuhochdeutschen. Zur endgültigen Grammatikalisierung der tun-Periphrase kommt es demnach im Standarddeutschen nicht ${ }^{3}$.

Die Grammatikalisierung vom Verb tun bzw. ihre Versuche finden sich auch in anderen Sprachen, z. B. im Englischen (do-Konstruktionen) und Niederländischen (doen-Konstruktionen). Im Englischen hat do in Fragesätzen und negierten Sätzen eine obligatorische Hilfsverbfunktion mit einem syntaktischen Zweck (Do you live in London? I don't live in London.). Im nicht negierten indikativen Satz dient hingegen do der Betonung des Sachverhalts (I do live in London.) (Huddleston 1984, S. 138). Die Umschreibung mit do hat sich im Englischen um 1700

${ }^{2} \mathrm{Zu}$ alternativen Hypothesen zur Entstehung des Dentalsuffixes als Präteritummarkers bei schwachen Verben vgl. Woźnicka 2017, S. 55-63.

${ }^{3}$ Die Entwicklung der tun-Periphrase, ihr Gebrauch und ihre Funktion im Deutschen, in seinen Dialekten wie auch im Englischen, Niederländischen und Italienischen wird in Schwarz (2009) detailliert beschrieben. 
fest verankert, wahrscheinlich wegen der mangelnden Flexionsendungen im englischen Präsens und Präteritum (Kortmann 2004, S. 245-276). Im Niederländischen dagegen gehört die Umschreibung mit doen, wie auch im Deutschen, nicht der Standradsprache und wird als die von ungebildeten Sprechern benutzte Konstruktion stigmatisiert (Langer 2001, S. 18).

\section{Materialbasis, Korpus, Methode}

Das Ziel des vorliegenden Beitrags ist die Beschreibung der formalen Entwicklung vom Verb tun, das im Gegenwartsdeutschen wegen der Stammvokalalternation (tun-tat-getan) als unregelmäßig bzw. stark betrachtet wird. Dabei werden jeweils die Formen des Vollverbs in synthetischen Konstruktionen der Analyse unterzogen, d. i. im Präsens (Indikativ, Optativ/Konjunktiv, Imperativ) und Präteritum (Indikativ und Optativ/Konjunktiv). Die Untersuchung erfolgt anhand eines abgegrenzten Textkorpus für das Alt- (http://titus.uni-frankfurt.de/indexe.htm), Mittel- (https://www. linguistics.ruhr-uni-bochum.de/rem/) und Frühneuhochdeutsche (https://korpora. zim.uni-duisburg-essen.de/Fnhd/). Im Falle des Althochdeutschen wird die Analyse grundsätzlich auf fünf Texte (Interlinearversionen der Benediktinerregel, St. Gallen (B) und der Murbacher Hymne, Reichenau, Murbach (H), Tatian, Fulda (T), Otfrids Evangelienbuch, Weißenburg, um 900 in Freising bair. umgeschrieben (O) und das herausragende Werk Notkers des Deutschen, St. Gallen, $\uparrow 1022(\mathbf{N}))$, die zwischen dem 9. und 11. Jahrhundert entstanden sind, beschränkt. Berücksichtigt werden jedoch auch die ältesten Formen, die nicht immer belegt sind und somit auf der Basis der belegten jüngeren Formen infolge des Vergleichs mit Formen anderer Verben abgeleitet werden. Die Formen werden den althochdeutschen Glossen (Abrogans) aus der 1. Hälfte des 8. Jahrhunderts aus dem angelsächsischen Missionsgebiet entnommen. Im Bezug auf zwei weitere Entwicklungsetappen des Deutschen werden alle im Rahmen des jeweiligen Korpus bearbeiteten Texte in Betracht gezogen. Die morphologische Analyse basiert im Prinzip auf dem von Darski (1987, 22004, 2010) vorgeschlagenen Analysemodell, dass im Rahmen des Beitrags wegen seines synchronen Charakters an die diachrone Herangehensweise angepasst wird.

\section{Die althochdeutschen Formen des Verbs tun}

Die Flexion im Präsens divergiert je nach der Quelle. Die Vielfalt der Formen resultiert daraus, dass:

1. Der Vokal /o:/ der ältesten Form tōn zunächst zu /uo/, /ua/, /oa/ diphthongiert wurde (die sog. althochdeutsche Diphthongierung der germanischen Langvokalen /o:/ i /e:/), wobei der Diphthong /oa/ ausschließlich in Singularformen 
des Präsens Indikativ (toam, ich tue ${ }^{6}$, toas , du tust', toat , er tut ${ }^{\star}$ ) und in der 3. P. Pl. (toant, sie tun') in Pariser und St. Galler Handschrift des Abrogans festgestellt werden (Braune, Reiffenstein 2004, S. 309);

2. Nach Analogie der starken Verben oft Formen mit Flexionsvokal auftreten, z. B. tüist, duis( $(t)$,du tust', duit ,er tut', tûên, tuoên ,wir tun', duet ,ihr tut', duent ,sie tun', tüe, tuoe, due, tûe, tuoe ,ich tue' (Konj.) (ebenda);

3. Vor dem Flexions-/Themavokal der zweite Vokal des Diphthongs i. d. R. verschwindet und der $u$-Vokal zu /u:/ gedehnt wird. Dabei wird /u:/ mit dem folgenden Thema-/Flexionsvokal nicht als Diphthong betrachtet, sondern beide bilden getrennte Silben. Von der Länge des Vokals zeugt der Zirkumflex über dem $u$-Vokal bei Notker, z. B. tûêst, du tuest' (2. P. Sg. Opt. Präs.). In Otfrids Evangelienbuch sind allerdings /ue/ und /ui/ Diphthonge, da beide metrisch als einsilbig verwendet werden. Nach Willmanns (1889, S. 424-426) haben sich diese Formen sekundär dem einsilbigen Grundtyp des Verbs tuon angeglichen.

Darüber hinaus vermischt sich die Flexion im Präsens vielfach mit Präsensformen der schwachen Verba pura auf uo, z. B. bluoen ,blühen', gruoen, wachsen“ (Knapp 1968, S. 301-303; Seebold 1970, S. 157-159; Lühr 1984, S. 42-44; Matzel 1987, S. 199-201).

Präsens

\begin{tabular}{|c|c|c|c|c|c|c|c|}
\hline \multirow[t]{7}{*}{ Indikativ } & & \multicolumn{2}{|c|}{$\begin{array}{l}\text { Die älteste Form } \\
\text { tōn }\end{array}$} & \multirow{2}{*}{$\begin{array}{l}\mathrm{B}, \mathrm{H} \\
\text { tuan } \\
\text { tuam }\end{array}$} & \multirow{2}{*}{$\begin{array}{l}\mathrm{T} \\
\text { tuon } \\
\text { tuon }\end{array}$} & \multirow{2}{*}{$\begin{array}{l}\mathrm{O} \\
\text { duan } \\
\text { duan }\end{array}$} & \multirow{2}{*}{$\begin{array}{l}\mathrm{N} \\
\text { tûon } \\
\text { tuon }\end{array}$} \\
\hline & Sg. & 1. & tōm & & & & \\
\hline & & 2. & tōs & - & $\begin{array}{l}\text { tuos, tuost } \\
\text { (tūis) }\end{array}$ & $\begin{array}{l}\operatorname{duas}(\mathrm{t}), \\
\operatorname{duis}(\mathrm{t})\end{array}$ & tuost \\
\hline & & 3. & tōt & tuat & tuot & duat, duit & tuot \\
\hline & Pl. & 1. & tōmēs & tuamēs & $\begin{array}{l}\text { tuomēs, } \\
\text { tuon }\end{array}$ & duen & $\begin{array}{l}\text { tûên, } \\
\text { tuoên }\end{array}$ \\
\hline & & 2. & tōt & tuat & tuot & duet & tuont \\
\hline & & 3. & tōnt & tuant & tuont & $\begin{array}{l}\text { duent, } \\
\text { (duant) }\end{array}$ & tuont \\
\hline \multicolumn{8}{|l|}{ Optativ } \\
\hline & Sg. & $1 ., 3$. & tō & tūe & $\begin{array}{l}\text { tuo, (tuoe, } \\
\text { tuoa, tue) }\end{array}$ & due & tûe, tuoe \\
\hline & & 2. & tōs & tūēs & tues & duest & $\begin{array}{l}\text { tûêst, } \\
\text { tuoêst }\end{array}$ \\
\hline & Pl. & 1. & tōm & - & - & duen & $\begin{array}{l}\text { tûên, } \\
\text { tuoên }\end{array}$ \\
\hline & & 2. & tōt & tūēt & tuot & - & $\begin{array}{l}\text { tûênt, } \\
\text { tuoênt }\end{array}$ \\
\hline & & 3. & tō & tūēn & tuon & - & $\begin{array}{l}\text { tûên, } \\
\text { tuoên }\end{array}$ \\
\hline
\end{tabular}


Imperativ

$\begin{array}{lllllll}\text { Sg. } & 2 . & \text { tō } & \text { tua } & \text { tuo } & \text { dua } & \text { tuo } \\ \text { Pl. } & 1 . & \text { tōmēs } & \text { tuamēs } & \text { tuomēs } & \text { duemēs } & - \\ & \text { 2. } & \text { tōt } & \text { tuat } & \text { tuot } & \begin{array}{l}\text { duet, } \\ \text { (duat) }\end{array} & \text { tuont } \\ & & & & & & \end{array}$

Die Präteritumformen (1. und 3. P. Sg.) zeigen Reduplikationssilbe të-, während der Stamm und die Flexionsendung die Nebensilbe -ta bilden. Das sind erhaltene Reste der Reduplikation im deutschen Präteritum (vgl. griech. té-thēka). Auf diese Art und Weise wird noch das Präteritum einer Gruppe von gotischen Verben gebildet, z. B. haita, ich heiße' und haihait, ich hieß', wobei der Vokal der Reduplikationssilbe kurzes $/ \varepsilon /$ ist. Unverkennbar ist der kurze $e$-Vokal in Formen der 1. und 3. P. Sg. Die anderen Formen sind den Präteritalformen der starken Verben der V. Klasse angeglichen, z. B. gëban: gab ,ich/ er gab', gābum, wir gaben' und tāti , du tat(e)st', tātum/tâtun, wir taten', tātut ,ihr tatet', tātun , sie taten'. Nach Krause/Jankuhn (1966, Nr. 157; alem. ca 600) erweist die Präteritalform der 3. P. Pl. dedun , sie taten' in der Runeninschrift von Schretzheim noch den kurzen Reduplikationsvokal im Plural (Lühr 1984, S. 49-50).

Präteritum Indikativ

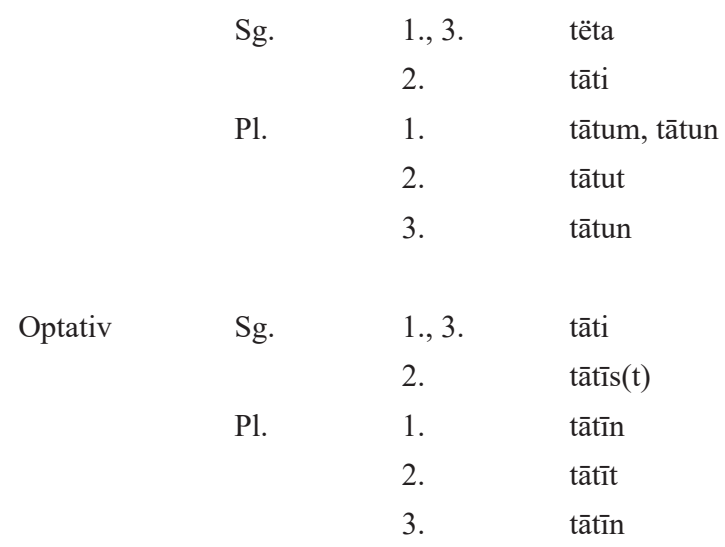

\section{Die mittelhochdeutschen Formen des Verbs tun}

Als Formen der 1. P. Sg. Präs. Ind. treten tuon und tuo ,ich tu(e) ${ }^{`}$ auf. Die ältere Form tuon zeigt die Primärendung - mi, die Form tuo ist dagegen Ergebnis der Angleichung an die Flexion der starken und schwachen Verben. Als Konkurrenzformen treten beide bei Hartmann von Aue (v. Kraus 1898, S. 155). 
Die mittelfränkischen Formen deist , du tust' und deit, er tut entstanden nach Analogie zu athematischen Verben gân/gên, gehen' und stân/stên ,stehen', in denen der lange Vokal /a:/ bzw. /e:/ (gâst/gêst, gât/gêt und stâst/stêst, stât/stêt), durch ei ersetzt wird (Übertritt in die thematische Flexion): geist , du gehst", geit ,er geht ${ }^{6}$; steist , du stehst', steit ,er steht" (Boesch 1946, S. 200).

Alemannische Konjunktivformen tüeje, tüege, auch: tuoge, tuohe sind analog $\mathrm{zu}$ Formen der ahd. schwachen $\bar{o} n$-Verben entstanden. Die Formen mit $j$ bzw. $g$, die schon in ahd. Texten auftreten, z. B. tuoie, tuoiest, tuoge (Kögel 1884, S. 509), sind Ergebnis des Einschubs in den Inlaut nach langen Vokalen und Diphthongen.

Im Präteritum ist die Silbe të- in tëte Reduplikationssilbe, während Stamm und Endung zu -te- geschwächt sind. Die Form tete mit gespanntem /e/ ist bei Gottfried von Straßburg (neben tëte), Konrad von Würzburg, Reinbot von Durne, Hugo von Trimberg u. a. zu finden. Die einsilbigen Formen der 1. und 3. P. Sg. (tët, tet) erscheinen auch bei Dichtern, die sonst das auslautende - $e$ beibehalten, z. B. Rudolf von Ems. Die Form tete ist dabei durch Enklise mit Personalpronomen ich aus der Form tëtich entstanden, in der $i$ des Personalpronomens den Umlaut hervorruft (Paul 2007, S. 73).

Die Singular- und Pluralparadigmen zeigen gegenseitig beeinflusste Formen:

1. tëten ist selten auftretende Pluralform, die analog zum Singular (tëte) gebildet wird;

2. tate ist gelegentlich vorkommende Singularform nach dem Plural tâten.

Die Pluralformen des Indikativs mit umgelautetem Stammvokal (tæten) gelten als analoge Bildungen zu Konjunktivformen, die sich beispielsweise in den ältesten Parzivalhandschriften finden, auch im Falle anderer Verben: wæren, bræ $h$ te. In Analogie zu Konjunktivformen tritt ebenso der Umlaut im Infinitiv und in präsentischen Pluralformen der sog. Präterito-Präsentien auf (Zwierzina 1900, S. 101, 255; Schirokauer 1923, S. 37). Die Form tëte im Konjunktiv gilt dagegen als analoge Bildung zu indikativischen Formen.

Präsens

$\begin{array}{llll}\text { Indikativ } & \text { Sg. } & 1 . & \text { tuon, tuo } \\ & & 2 . & \begin{array}{l}\text { tuos }(\mathrm{t}), \mathrm{mfrk} \text {. deis(t) } \\ \text { tuot, mfrk. deit }\end{array} \\ & \text { Pl. } & 1 . & \begin{array}{l}\text { tuon, alem. tuont } \\ \text { tuot, alem. tuont } \\ \end{array} \\ & & 2 . & \text { tuont } \\ \text { Konjunktiv } & \mathrm{Sg} . & 1 . & \text { tuo alem. tüeje } \\ & & 2 . & \begin{array}{l}\text { tuost, tuogest, tüejest } \\ \text { tuo, tüeje }\end{array}\end{array}$




\begin{tabular}{|c|c|c|c|}
\hline & Pl. & 1. & tuon, tüejen \\
\hline & & 2. & tuot, tüejet \\
\hline & & 3. & tuon, tüejen \\
\hline Imperativ & Sg. & 2. & tuo \\
\hline & Pl. & 1. & tuon \\
\hline & & 2. & tuot \\
\hline
\end{tabular}

Präteritum

\begin{tabular}{|c|c|c|c|}
\hline \multirow[t]{6}{*}{ Indikativ } & \multirow[t]{3}{*}{ Sg. } & 1. & tët(e), tet( \\
\hline & & 2. & tæte \\
\hline & & 3. & tët(e), tet( \\
\hline & \multirow[t]{3}{*}{ Pl. } & 1. & tâten, tæt \\
\hline & & 2. & tâtet \\
\hline & & 3. & tâten \\
\hline \multirow[t]{6}{*}{ Konjunktiv } & \multirow[t]{3}{*}{ Sg. } & 1. & tæte, tëte \\
\hline & & 2. & tætest \\
\hline & & 3. & tæte \\
\hline & \multirow[t]{3}{*}{ Sg. } & 1. & tæten \\
\hline & & 2. & tætet \\
\hline & & 3. & tæten \\
\hline
\end{tabular}

\section{Die frühneuhochdeutschen Formen des Verbs tun}

Das Verb tun mit zahlreichen Nebenformen (dem anlautenden $d$-, verschiedenen stammvokalischen Veränderungen u. a: $a, o, u o, a i, \ddot{u}$ wie auch zweisilbigen Formen: tüejen, tuenen, tüenen) weist flexivische Besonderheiten des Präsens und des Präteritums auf, die noch im Verlauf des Frühneuhochdeutschen größtenteils aufgehoben werden.

Das - $n$ der 1. P. Sg. im Präsens verschwindet, und das Verb wird an die Formen der starken und schwachen Verben angepasst; im Präteritum wird hingegen die Stammvokal-Alternation $e-a$ zugunsten $a$ angeglichen.

Präsens

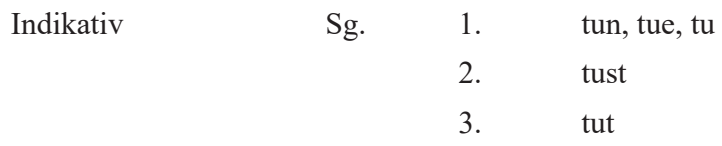




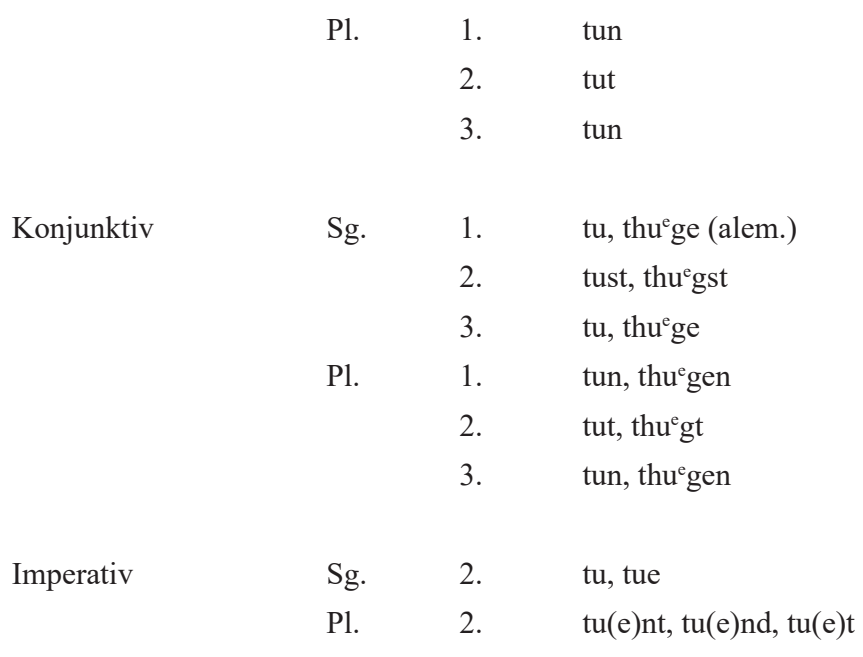

Präteritum

\begin{tabular}{|c|c|c|c|}
\hline \multirow[t]{6}{*}{ Indikativ } & Sg. & 1. & tet, tat \\
\hline & & 2. & tetest, tatets \\
\hline & & 3. & tet, tat \\
\hline & $\mathrm{Pl}$. & 1. & $\operatorname{taten}(\mathrm{t})$, teten $(\mathrm{t})$ \\
\hline & & 2. & tatet, $\operatorname{taten}(\mathrm{t})$, tetet, teten $(\mathrm{t})$ \\
\hline & & 3. & $\operatorname{taten}(\mathrm{t}), \operatorname{teten}(\mathrm{t})$ \\
\hline
\end{tabular}

Das $-n$ der 1. P. Sg. Ind. Präs. erscheint bis in das 16. Jahrhundert. Als Nebenform gilt tu, die seit dem 14. Jahrhundert vorwiegend im Konjunktiv auftritt. Spätestens im 17. Jahrhundert ist die Form tue fest.

Auf dem oberdeutschen Sprachgebiet hält der Singular -e- bis um 1500 fest (Strömberg 1907, S. 137), angeglichene Formen mit - $a$ - sind allerdings möglich, z. B. tet vs. tat ,ich/er tat', tetest vs. tatest, du tatest'. Schon im 14. Jahrhundert, also früher als Singularformen zeigen Pluralformen das $-e$ - des Singulars (Strömberg 1907, S. 138), z. B. taten $(t)$ vs. teten $(t)$, wir/ihr/sie taten/tatet', tatet vs. tetet ,ihr tatet'. Im 16. Jahrhundert konkurrieren miteinander Formen mit $-a$ - und -e(Strömberg 1907, S. 127, 130, 132, 135), wobei die $e$-Formen auch in der 2. Hälfte des 16. Jahrhunderts die häufigeren sind. Bis zum Ende des 16. Jahrhunderts vermutet Strömberg (1907, S. 137f.) die Festigung der $a$-Formen im Plural sowie ihre Durchsetzung im Singular.

Schon im 14. Jahrhundert beobachtet Alm (1936) im Ostmitteldeutschen den Ausgleich in beiden Richtungen. Im 15. Jahrhundert überwiegen $a$-Formen sowohl im Singular als auch im Plural (Alm 1936, S. 439). Den Umschwung, d. i. die Festigung der $e$-Formen im Singular und ihr Eindringen in den Plural erklärt 
Alm (1936, S. 439) als Einfluss des Oberdeutschen. Die herandringenden Formen that, thaten tragen allerdings zur allmählichen Entwicklung zugunsten der $a$-Formen im Präteritum bei. Abgeschlossen wird der Prozess bis zum Ende des 17. Jahrhunderts, wobei er zuerst im Oberdeutschen, dann im Thüringischen, Norddeutschen und schließlich im Schlesischen stattfand (ebenda).

\section{Fazit}

Das Ziel des Beitrags war es, differente Formen des Verbs tun anhand der Korpusanalysen und -daten zu ermitteln und ihre Entwicklung bis zum Neuhochdeutschen hin zu beschreiben. Die Formen des Verbs tun variieren dabei infolge zahlreicher, von der Entwicklungsetappe wie auch dem Paradigma abhängiger Prozesse. Die breite Palette von tun-Formen beweist die Anpassungsfähigkeit des Verbs zu zwei Hauptklassen, d. i. den starken und/oder schwachen Verben bzw. deren Subklassen. Besonders im Frühneuhochdeutschen sind hingegen Ausgleichsprozesse zwischen Formen innerhalb eines Paradigmas wie auch verschiedener Paradigmen sichtbar (inter- und intraparadigmatischer Ausgleich), so dass zum Ausgang des 17. Jahrhunderts die Formen des Verbs im Rahmen aller Paradigmen weitgehend gefestigt sind.

\section{LITERATURVERZEICHNIS}

Alm E. (1936), Der Ausgleich des Ablauts im starken Czas przeszty der ostmitteldeutschen Schriftdialekte: I. 1.-3. Ablautreihe und das Verb tun, Uppsala: Appelbergs boktryckeriaktiebolag.

Bammesberger A. (1986), Der Aufbau des germanischen Verbsystems, Heidelberg: Winter.

Boesch B. (1946), Untersuchungen zur alemannischen Urkundensprache des 13. Jahrhunderts. Laut- und Formenlehre, Bern: A. Francke.

Bopp F. (1816), Das Konjugationssystem der Sanskrit-Sprache in Vergleichung mit jenem der griechischen, lateinischen, persischen und germanischen Sprache, Frankfurt am Main: Athenäum.

Braune W., Reiffenstein I. (2004), Althochdeutsche Grammatik I. Laut- und Formenlehre. 15. Auflage. Tübingen: Max Niemeyer Verlag.

Collitz H. (1888), Die Herkunft des schwachen Präteritums der germanischen Sprachen. In: American Journal of Philology 44, S. 42-57.

Collitz H. (1912), Das schwache Präteritum und seine Vorgeschichte, Göttingen: Vandenhoeck \& Ruprecht.

Darski J. (1987), Linguistisches Analysemodell. Definitionen grundlegender grammatischer Begrif$f e$, Poznań: Wydawnictwo Naukowe UAM.

Darski J. (2004), Linguistisches Analysemodell. Definitionen grundlegender grammatischer Begrif$f e .2$., völlig neu bearbeitete und ergänzte Auflage. Poznań: Wydawnictwo Naukowe UAM.

Darski J. (2010), Deutsche Grammatik. Ein völlig neuer Ansatz, Frankfurt am Main-Berlin-BernBruxelles-New York-Oxford-Wien: Peter Lang Verlag.

Deuber C., Bürgisser U., Bajna E. (2007), Protokoll der Sitzung vom 12. Dezember 2007, Fallbeispiel: am-Progressiv und tun-Periphrase, Universität Zürich. 
DUDEN. Das große Wörterbuch der deutschen Sprache (1999), 4., vollständig überarbeitete Auflage. Herausgegeben von der Dudenredaktion. Projektleitung: Dr. Werner Scholze-Stubenrecht, Mannheim-Leipzig-Wien-Zürich: Dudenverlag.

DUDEN 4. Die Grammatik (2001), Mannheim-Leipzig-Wien-Zürich: Dudenverlag.

DUDEN 4. Die Grammatik. (2005), 7., völlig neu erarbeitete und erweiterte Auflage. Herausgeben von der Dudenredaktion. Bearbeitet von Peter Eisenberg, Jörg Peters, Peter Gallmann, Catherine Fabricius-Hansen, Damaris Nübling, Irmhild Barz, Thomas A. Fritz, Reinhard Fiehler, Mannheim-Leipzig-Wien-Zürich: Dudenverlag.

Ebert R.P., Reichmann O., Solms H.-J., Wegera K.-P. (1993), Frühneuhochdeutsche Grammatik. Hrsg. von Oskar Reichmann, Klaus-Peter Wegera. Max Niemeyer Verlag: Tübingen.

Eichinger L.M. (2014), Trennbare Verben und Grammatikalisierung: feindliche Brüder? In: Schriften des Instituts für Deutsche Sprache. https://de.scribd.com/document/322185625/Eichinger-Trennbare-Verben-Und-Grammatikalisierung-2004 (Zugriff am: 27.10.2018).

Engel U. (1996), Deutsche Grammatik, Heidelberg: Groos.

Grimm J. (1989), Deutsche Grammatik (1819-1831), Neuer vermehrter Abdruck, Gütersloh: Druck und Verlag von C. Bertelsmann 1898, Neuauflage: Leipzig, Enzyklopädie.

Hirt H. (1927), Indogermanische Grammatik. Teile I-VII, Heidelberg: Winter.

Huddleston R. (1984), Introduction to the Grammar of English, Avon: Cambridge University Press.

Knapp F.P. (1968), Ahd. teta - tātum. In: Birkhan Helmut, Gschwantler Otto (Hrsg.), Festschrift Otto Höfler, Wien: Notring, S. 301-314.

Kögel R. (1884), Die schwachen Verba zweiter und dritter Klasse. In: Paul und Braunes Beiträge zur Geschichte der deutschen Sprache und Literatur 9. Halle/Salle 1874-1954, S. 504-523.

Kortmann B. (2004), DO as a tense and aspect marker in varieties of English. In: Kortmann Bernd (Hrsg.), Dialectology meets typology. Dialect grammar from a cross-linguistic perspective, Berlin-New York: Walter de Gruyter, S. 245-276.

Krause W., Jankuhn, H. (1966), Die Runeninschriften im älteren Futhark, Göttingen: Vandenhoeck $\&$ Ruprecht.

Langer N. (2001), Linguistic purism in Action: How auxiliary, tun ' was stigmatized in Early New High German, Berlin-New York: Walter de Gruyter.

Lühr R. (1984), Reste der athematischen Konjugation in den germanischen Sprachen. In: Utermann Jürgen, Brogyanyi Bela (Hrsg.), Das Germanische und die Rekonstruktion der idg. Grundsprache. Amsterdam (Amsterdam Studies in the Theory and History of Linguistic Science, Ser. IV: Current Issues in Linguistik Theory, 22), S. 35-90.

Matzel K. (1987), Zu den verba pura des Germanischen. Kuhns Zeitschrift für vergleichende Sprachforschung 100, S. 146-203.

Paul H. (2007), Mittelhochdeutsche Grammatik. 25. Auflage, neu bearbeitet von Thomas Klein, Hans-Joachim Solms und Klaus-Peter Wegera. Mit einer Syntax von Ingeborg Schöbler, neubearbeitet und erweitert von Heinz-Peter Prell, Tübingen: Max Niemeyer Verlag.

Schirokauer A.C. (1923), Studien zur mittelhochdeutschen Reimgrammatik. Paul und Braunes Beiträge zur Geschichte der deutschen Sprache und Literatur, 47, S. 1-126.

Schwarz C. (2009), Die ,tun'-Periphrase im Deutschen: Gebrauch und Funktion, Wien: VDM Verlag.

Seebold E. (1970), Vergleichendes und etymologisches Wörterbuch der germanischen starken Verben. Den Haag (Janua Linguarum. Series pracitca 85).

Strömberg E. (1907), Die Ausgleichung des Ablauts im starken Präteritum; mit besonderer Rücksicht auf oberdeutsche Sprachdenkmäler des 15.-16. Jahrhunderts, Göteborg: W. Zachrissons boktryckeri a.-b.

Sverdrup J. (1929), Das germanische Dentalpräteritum. Norsk tidskrift for sprogvidenskap 2, S. 5-96. 
von Kraus C. (1898), Das sogenannte II. Büchlein und Hartmanns Werke. In: Abhandlungen zur Germanischen Philologie. Fs. R. Heinzel. Halle/S. (Unveränderter reprographischer Nachdruck Hildesheim, Zürich, New York 1985, S. 111-172).

Weiss E. (1956), Tun: machen. Bezeichnungen für die kausative und periphrastische Funktion im Deutschen bis um 1400, Stockholm: Acta Universität.

Willmanns W. (1889), Die Flexion der Verben , tuon', ,gân', ,stân 'im Ahd, Zeitschrift für deutsches Altertum und deutsche Literatur 33, S. 424-431.

Woźnicka M. (2017), Zur Herkunft und Entwicklung des Präteritummarkers. In: Woźnicka Marta, Stolarczyk-Gembiak Anna (Hrsg.), Annäherungen 2. Sprachwissenschaft - Literaturwissenschaft, Konin: Państwowa Wyższa Szkoła Zawodowa, S. 55-63.

Zwierzina K. (1900), Mittelhochdeutsche Studien. Zeitschrift für deutsches Altertum und deutsche Literatur, 44, S. 1-116.

\title{
Korpora/Quellenverzeichnis
}

Althochdeutsch: http://titus.uni-frankfurt.de/texte/etcs/germ/ahd/tatian/tatia.htm (Zugriff am: 27.10.2018).

Frühneuhochdeutsch: Das Bonner Frühneuhochdeutsch-Korpus Korpora.org, http://www.korpora. org/fnhd/ (Zugriff am: 27.10.2018).

Mittelhochdeutsch: Klein Thomas; Wegera Klaus-Peter; Dipper Stefanie; Wich-Reif Claudia (2016). Referenzkorpus Mittelhochdeutsch (1050-1350), Version 1.0, https://www.linguistics. ruhr-uni-bochum.de/rem/. ISLRN 332-536-136-099-5 (Zugriff am: 27.10.2018).

Pariser Handschrift des Glossars Abrogans 1, 2-198,10. Vollfaks.: Baesecke 1926, S. 1-20.

St. Galler Handschrift des Glossars Abrogans („Keronisches Glossar“). BV 253. Vollfaks.: Bischoff/ Duft/Sonderegger 1977; Fischer ST 1a.

Marta Woźnicka

\begin{abstract}
FORMAL DEVELOPMENT OF THE VERB TUN ("DO, MAKE")
IN THE GERMAN LANGUAGE A CORPUS INVESTIGATION FROM THE OLD TO THE MODERN-NEW-HIGH-GERMAN STAGE
\end{abstract}

(Summary)

The article aims to introduce the formal development of the verb tun ("do, make") in the German language, based on the corpuses of old (http://titus.uni-frankfurt.de/indexe.htm), middle (https:// www.linguistics.ruhr-uni-bochum.de/rem/) and modern-new-high-German language (https://korpora. zim.uni-duisburg-essen.de/Fnhd/). However, the morphological analysis is primarily based on Józef Darski's $(1987,2004,2010)$ innovative model of linguistic analysis which, due to its synchronic character, has been adopted in diachronic research. The verb forms of tun, prone to modifications owing to multiple processes depending on both the stage of development and the language as a paradigm, reveal its capability of adaptation to the two basic classes of verbs, i.e. strong verbs and/or weak verbs and their subclasses. Additionally, it is possible to observe the processes of homogenization of the verb forms within a given paradigm and among the forms of distinct paradigms, mainly to stabilise them.

Keywords: verb tun, old-high-German, middle-high-German, early-new-high-German, formal development 